\title{
artigo
}

Hodniki, P.P.; Teixeira, C.R.S.; Zanetti, M.L.; Moraes, C.; Fermino, R.C.; Berra, S.

Prática de atividade física e ambiente percebido de usuários do Sistema Único de Saúde

\section{Prática de atividade física e ambiente percebido de usuários do Sistema Único de Saúde}

\author{
Practice of physical activity and perceived environment of users of the Unified Health System \\ Práctica de actividad física y ambiente nfermida de nfermid del Sistema Único de Salud
}

\begin{abstract}
RESUMO
Objetivo: analisar a relação da atividade física no lazer e de deslocamento ativo com a percepção do ambiente em pessoas com doenças crônicas não transmissíveis. Métodos: estudo transversal constituído por 719 usuários do SUS. Para a coleta de dados utilizou-se o IPAQ e o Neighborhood Environmental Walkability Scale. Utilizou-se o teste qui-quadrado de Pearson e o modelo de regressão logística. Resultados: dos $719(100 \%), 512(71,2 \%)$ participantes eram do sexo feminino e 504 (70,1\%) relataram ter doença crônica não transmissível. Não há relações entre mulheres com doença crônica e percepção do ambiente para a prática de atividade física no lazer e de deslocamento ativo. Por outro lado, os homens e os idosos apresentaram maior chance de serem mais ativos para a prática de atividade física. Conclusão: intervenções para incrementar a prática de atividade física em mulheres devem ser implementadas com vistas a prevenção e tratamento das doenças crônicas não transmissiveis.
\end{abstract}

DESCRITORES: Atividade Física; Doenças Crônicas; Ambiente; Sistema Único de saúde.

\section{ABSTRACT}

Objective: to analyze the relationship between leisure-time physical activity and active commuting with the perception of the environment in people with chronic non-communicable diseases. Methods: cross-sectional study consisting of 719 SUS users. For data collection, the IPAQ and the Neighborhood Environmental Walkability Scale were used. Pearson's chi-square test and logistic regression model were used. Results: of the $719(100 \%), 512(71.2 \%)$ participants were female and $504(70.1 \%)$ reported having a chronic non-communicable disease. There are no relationships between women with chronic disease and perception of the environment for the practice of physical activity during leisure and active commuting. On the other hand, men and the elderly were more likely to be more active in the practice of physical activity. Conclusion: interventions to increase the practice of physical activity in women should be implemented in order to prevent and treat chronic non-communicable diseases.

DESCRIPTORS: Physical activity; Chronic diseases; Environment; Health Unic System.

\section{RESUMEN}

Objetivo: analizar la relación entre la actividad física com el nfer libre y los desplazamientos activos com la percepción del entorno com personas com nfermidades crónicas no transmisibles. Métodos: nfermi transversal compuesto por 719 usuarios del SUS. Para la recolección de datos, se utilizaron el IPAQ y la Escala de Caminabilidad Ambiental del Vecindario. Se nfermi la prueba de chi-cuadrado de Pearson y el modelo de regresión logística. Resultados: de los $719(100 \%), 512$ (71,2\%) participantes eran mujeres y $504(70,1 \%)$ informaron tener com enfermedad crónica no transmisible. No existen relaciones entre mujeres com enfermedad crónica y percepción del entorno para la práctica de actividad física durante el nfe y los desplazamientos activos. Por com lado, los hombres y los ancianos tenían más probabilidades de ser más activos com la práctica de actividad física. Conclusión: se deben implementar intervenciones para incrementar la práctica de actividad física com las mujeres com el fin de prevenir y tratar las nfermidades crónicas no transmisibles.

DESCRIPTORES: Actividad física; Enfermedades crónicas; Ambiente; Sistema único de Salud.

RECEBIDO EM: 28/01/2021 APROVADO EM: 17/02/2021

\section{Paula Parisi Hodniki}

Pesquisadora, profissional de educação física, doutora em ciência pela Universidade de São Paulo, Escola de Enfermagem de Ribeirão Preto, Departamento de Enfermagem Geral e Especializada.

ORCID: 0000-0003-1643-2134 


\section{Carla Regina de Souza Teixeira}

Docente, doutora em ciência pela Universidade de São Paulo, Escola de Enfermagem de Ribeirão Preto, Departamento de Enfermagem Geral e Especializada.

ORCID: 0000-0002-8887-5439

\section{Maria Lúcia Zanetti}

Docente, doutora em ciência pela Universidade de São Paulo, Escola de Enfermagem de Ribeirão Preto, Departamento de Enfermagem Geral e Especializada.

ORCID: 0000-0002-8887-5439

\section{Camila de Moraes}

Docente, Universidade de São Paulo, Escola de Educação Física e Esporte de Ribeirão Preto, São Paulo, doutora. ORCID: 0000-0003-3735-1165

\section{Rogério Cesar Fermino}

Docente, Universidade Tecnológica Federal do Paraná, doutor.

ORCID: 0000-0002-9028-4179

\section{Silvina Berra}

Docente, Universidad Nacional de Córdoba, Córdoba, Argentina.

ORCID: 0000-0003-3618-7041

\section{INTRODUÇÃO}

A prática regular de atividade física é uma das ações estratégicas para a prevenção das doenças crônicas não transmissíveis (DCNT) e de suas complicações, considerado um indicador importante para o monitoramento e avaliação de efetividade nas estratégias de promoção a saúde pela Política Nacional de Promoção da Saúde. Mesmo uma quantidade mínima de atividade física tem um efeito protetor na saúde contra doenças crônicas e reduz mortalidade. Evidências mostram redução de risco de mortalidade prematura em pelo menos $20,0 \%$ a $30,0 \%$ para mais de 25 tipos de doenças crônicas ${ }^{1,2}$.

Entretanto, apenas $38,1 \%$ da população brasileira adulta cumpre o mínimo preconizado pela Organização Mundial da Saúde quanto à frequência da prática de atividade física no tempo livre (lazer), o que equivale a 150 minutos de atividade moderada por semana. Em relação ao deslocamento ativo, apenas $14,4 \%$ dos brasileiros maiores de 18 anos realizam a atividade física por pelo menos 150 minutos por semana ${ }^{3}$. Pessoas com condições crônicas são capazes de sustentar a atividade física por mais de três meses, sob diferentes graus de supervisão pelos profissionais envolvidos, em níveis suficientes para a saúde3. Por outro lado, inatividade física, a dieta não saudável, o uso abusivo do álcool e tabagismo são fatores de risco comportamental para DCNT, pois, resultam em quadros de hiperglicemia, hipertensão, hipercolesterolêmica e excesso de peso corporal ${ }^{4}$.

O Plano de Ações Estratégicas para o Enfrentamento das DCNT no Brasil, 2011-2022, com a finalidade de promover o desenvolvimento de políticas públicas efetivas e sustentáveis para a prevenção e o controle das DCNT e seus fatores de risco, visa reduzir a morbidade, incapacidade e mortalidade, por meio de um conjunto de açóes preventivas e promocionais de saúde, reordenando os serviços de saúde do Sistema Único de Saúde (SUS), a partir da Atenção Primária e da participação comunitária 5 .

O ambiente interfere de forma positiva ou negativa no comportamento e estilo de vida da população, primordialmente no que concerne a adoção de hábitos de vida saudáveis ${ }^{6}$. Fatores ambientais afetam de maneira desigual os diferentes segmentos sociais da população ${ }^{6}$. Nesse contexto, o ambiente é considerado um dos fatores que levam a tomada de decisões na área da saúde.

Nesta direção, a literatura aponta in- tervenções que requerem implementação e monitoramento urgentes pelos gestores e políticas públicas ${ }^{7}$.

No Brasil, cabe destacar que o perfil dos usuários do SUS é de indivíduos com menor renda e escolaridade, pessoas com doenças crônicas, maioria mulheres e que refere buscar atendimento por motivo de doença/queixa ${ }^{8}$. Desse modo, investigar o perfil sociodemográficos e de saúde da população usuária dos serviços de saúde do SUS pode contribuir para fomentar estratégias futuras em relação a percepção do ambiente e AF de pessoas com DCNT.

Diante do exposto, este estudo tem como objetivo investigar a percepção do ambiente para a prática de atividade física e deslocamento ativo em pessoas com DCNT. Espera-se que o resultado desse estudo possa fornecer subsídios para direcionar políticas públicas no que tange a promoção da prática de atividade física como ação preventiva para o desenvolvimento de DCNT.

\section{MÉTOdOS}

Estudo quantitativo, descritivo, observacional e transversal, realizado em cinco Distritos de Saúde do município de Ribeirão Preto, SP, cuja população 


\section{artigo}

Hodniki, P.P.; Teixeira, C.R.S.; Zanetti, M.L.; Moraes, C.; Fermino, R.C.; Berra, S.

Prática de atividade física e ambiente percebido de usuários do Sistema Único de Saúde

segundo censo de 2010 é de 604.682 habitantes, considerado o oitavo município mais populoso do estado. ${ }^{9}$ É referência da região de saúde da Direção Regional de Saúde XIII (DRSXIII). ${ }^{10}$ A amostra foi calculada por amostragem aleatória estratificada, na qual cada estrato foi formado pelos distritos de saúde de Ribeirão Preto, SP. Adotando-se os parâmetros de erros relativos de amostragem de 10\%, nível de significância de 5\% e o número total da média mensal de atendimentos nas farmácias dos cinco distritos, no ano de 2016, obteve-se 53.240 atendimentos mensais. A amostra sobre AF foram obtidos a partir de um estudo piloto, realizado com 50 indivíduos, distribuídos igualmente nos cinco distritos de saúde. Foram adotados como referência os estudos com pessoas que avaliaram a percepção do ambiente para a prática de AF. A amostra foi constituída por 719 pessoas adultas ( $\geq 18$ anos), de ambos o sexo, atendidas nos cinco Distritos de Saúde de Ribeirão Preto, SP, e que referiram apresentar ou não DCNT (Tabela 1).

Foram eleitas as variáveis sociodemográficas: sexo, idade, escolaridade, ${ }^{11}$ ocupação, estado civil e classe econômica segundo a Associação Brasileira de Empresas de Pesquisa $(\mathrm{ABEP}) .^{12} \mathrm{Em}$ relação às variáveis clínicas, as doenças crônicas não transmissivas foram autorreferidas pelas pessoas e classificadas em sim/não. Considerou-se a hipertensão arterial sistêmica (HAS), acidente vascular cerebral (AVC), doença do coração (DC), Diabetes Mellitus Tipo 1 (DM1), Diabetes Mellitus Tipo 2 (DM2), câncer, rinite, sinusite, doença pulmonar obstrutiva crônica, asma e bronquite e outras doenças autorreferidas. ${ }^{3,13}$

Para obtenção dos dados referentes à atividade física utilizou-se o International Physical Activity Questionnaire (IPAQ), versão longa. O IPAQ tem como domínios atividade física de recreação, esporte, exercício e de lazer, denominado atividade física de lazer (AFL), e atividade física como meio de transporte, como forma de deslocamento (AFD). Para classificação do nível de atividade física foi utilizado a recomendação da Organização Mundial da Saúde (OMS), que considera ativos aqueles que realizam pelo menos 150 minutos de atividade física moderada na semana ou 75 minutos de atividade física vigorosa na semana. ${ }^{14}$ Para obtenção das características do ambiente percebido empregou-se a Neighborhood Environmental Walkability Scale (NEWS) adaptada, ${ }^{5,15}$ a qual permite avaliar a percepção do ambiente para a prática de atividade física em adultos e idosos. A primeira parte da NEWS adaptada é composta por 18 questóes referentes à acessibilidade a conveniência. Essas questões estão relacionadas ao tempo que o indivíduo levaria caminhando para chegar da sua residência até conveniências comerciais, de serviço ou de lazer no bairro onde residiam. A segunda parte é composta por 20 questões relacionadas à estrutura ambiental próxima da residência. Dessas, três questões referem-se a segurança no trânsito tais como trânsito intenso que pode dificultar a caminhada ou o uso de bicicleta, faixas de segurança para pedestres, respeito aos pedestres pelos motoristas nas faixas de segurança. ${ }^{5}$

A coleta de dados foi realizada no período de maio de 2017 a abril de 2018, por meio de entrevistas aos usuários do SUS de cada Distrito de Saúde do município. As questões estatisticamente significantes foram adicionadas a análise de regressão. Posteriormente foi utilizada regressão logística para analisar se houve diferença na prática de AFL e AFD com a percepção do ambiente em usuários do serviço público de saúde, e foi calculada a razão de Odds (OR). Foi utilizado intervalo de confiança a 95\%. Em todas as análises foi considerado um nível de significância de 5\% (alfa =0.05). As análises foram realizadas por meio do programa $\mathrm{R}$ versão 3.3.0. ${ }^{16}$

O projeto foi aprovado pelo Comitê de Ética em Pesquisa, parecer $n^{\circ}$ 1.875.599. O acesso aos Distritos de Saúde foi permitido mediante solicitação apresentada à Secretaria Municipal de Saúde do município. Os usuários do serviço de saúde assinaram um Termo de Consentimento Livre e Esclarecido para participação no estudo.

\section{RESULTADOS}

Dos 719 (100\%) usuários dos serviços públicos de saúde do município, maioria do sexo feminino. A média de idade foi de 44,83 anos, desvio padrão de ${ }^{17,64}$. Grande parte era casado ou em união estável. Parte significante tinha baixa escolaridade com

Tabela 1 - Número de participantes de cada Distrito de Saúde dos usuários do SUS segundo atendimentos mensais na farmácia (2016); estudo piloto; tamanho amostral e amostra do estudo Ribeirão Preto - SP, 2019

\begin{tabular}{lcccc}
\multicolumn{1}{c}{ DISTRITOS } & $\begin{array}{c}\text { ATENDIMENTOS } \\
\text { MENSAIS (2016) }\end{array}$ & ESTUDO PILOTO & $\begin{array}{c}\text { TAMANHO AMOSTRAL } \\
\text { (ERRO RELATIVO DE 10\%) }\end{array}$ & $\begin{array}{c}\text { AMOSTRA DO ESTUDO } \\
\text { (2017 E 2018) }\end{array}$ \\
Oeste & 7.015 & 10 & 84 & 107 \\
Central & 14.909 & 10 & 192 & 192 \\
Sul & 12.077 & 10 & 91 & 99 \\
Norte & 12.757 & 10 & 165 & 165 \\
Leste & 6.482 & 10 & 156 & 156 \\
Total & 53.240 & 50 & 688 & 719 \\
Fonte: elaboração própria & & & &
\end{tabular}


emprego ou eram aposentados ou pensionistas, a maioria era da classe $\mathrm{C}$, maioria referia que tinha DCNT (Tabela 2).

Quanto à percepção do ambiente e AFL dos usuários do SUS, foi constatado que os homens têm mais chance de serem ativos do que as mulheres (OR 1,55). Os participantes que residem longe de posto de saúde tem mais chance de serem mais ativos no lazer (OR 1,72). E os partici- pantes que não sabem ou disseram não ter feira no bairro onde residem, apresentaram o valor de chances opostas de serem ativos no lazer (OR 0,51) (Tabela 3). No que se refere aos resultados quanto à

Tabela 1 - Número de participantes de cada Distrito de Saúde dos usuários do SUS segundo atendimentos mensais na farmácia (2016); estudo piloto; tamanho amostral e amostra do estudo Ribeirão Preto - SP, 2019

\begin{tabular}{|c|c|c|c|c|c|c|c|}
\hline & & & & & & & \\
\hline & & & SIN & 504) & TOTAL & $N=719$ & P VALOR \\
\hline & N & $(\%)$ & N & $(\%)$ & N & $(\%)$ & \\
\hline Distritos de Saúde & & & & & & & $0,008^{*}$ \\
\hline Oeste & 19 & $(17,8)$ & 88 & $(82,2)$ & 107 & $(14,9)$ & \\
\hline Central & 60 & $(31,2)$ & 132 & $(68,7)$ & 192 & $(26,7)$ & \\
\hline Sul & 33 & $(33,0)$ & 67 & $(67,0)$ & 100 & $(13,9)$ & \\
\hline Norte & 62 & $(37,6)$ & 103 & $(62,4)$ & 165 & $(22,9)$ & \\
\hline Leste & 41 & $(26,4)$ & 114 & $(73,5)$ & 155 & $(21,6)$ & \\
\hline Sexo & & & & & & & 0,461 \\
\hline Feminino & 149 & $(29,1)$ & 363 & $(70,9)$ & 512 & $(71,2)$ & \\
\hline Masculino & 66 & $(31,9)$ & 141 & $(68,1)$ & 207 & $(28,8)$ & \\
\hline Idade - Média: 44,83 (17,64) & & & & & & & $<0,001^{*}$ \\
\hline 18 a 34 anos & 115 & $(47,5)$ & 127 & $(52,5)$ & 242 & $(33,7)$ & \\
\hline 35 a 59 anos & 76 & $(25,9)$ & 217 & $(74,1)$ & 293 & $(40,7)$ & \\
\hline 60 ou mais & 24 & $(13,0)$ & 160 & $(87,0)$ & 184 & $(25,6)$ & \\
\hline Estado Civil & & & & & & & $0,010^{*}$ \\
\hline Solteiro (a) & 98 & $(36,0)$ & 174 & $(64,0)$ & 272 & $(37,8)$ & \\
\hline Casado (a) ou união estável & 95 & $(28,0)$ & 244 & $(72,0)$ & 339 & $(47,1)$ & \\
\hline Viúvo & 6 & $(14,3)$ & 36 & $(85,7)$ & 42 & $(5,8)$ & \\
\hline Separado ou divorciado & 16 & $(24,2)$ & 50 & $(75,8)$ & 66 & $(9,9)$ & \\
\hline Escolaridade & & & & & & & $0,031^{*}$ \\
\hline Ensino Fundamental incompleto & 70 & $(23,9)$ & 223 & $(76,1)$ & 293 & $(40,7)$ & \\
\hline Ensino Fundamental completo & 17 & $(30,4)$ & 39 & $(69,6)$ & 56 & $(7,8)$ & \\
\hline Ensino Médio completo & 93 & $(35,1)$ & 172 & $(64,9)$ & 265 & $(36,9)$ & \\
\hline Ensino Superior & 35 & $(33,3)$ & 70 & $(66,7)$ & 105 & $(14,6)$ & \\
\hline Ocupação & & & & & & & $<0,001^{*}$ \\
\hline Com emprego & 107 & $(34,3)$ & 205 & $(65,7)$ & 312 & $(43,4)$ & \\
\hline Aposentado/Pensionista & 17 & $(10,2)$ & 150 & $(89,8)$ & 167 & $(23,2)$ & \\
\hline Desempregado & 91 & $(37,9)$ & 149 & $(62,1)$ & 240 & $(33,4)$ & \\
\hline Classe econômica & & & & & & & 0,203 \\
\hline$A+B$ & 85 & $(34,0)$ & 165 & $(66,0)$ & 250 & $(34,8)$ & \\
\hline C & 102 & $(27,3)$ & 271 & $(72,6)$ & 373 & $(51,9)$ & \\
\hline $\mathrm{D}+\mathrm{E}$ & 28 & $(29,2)$ & 68 & $(70,8)$ & 96 & $(13,3)$ & \\
\hline
\end{tabular}




\section{artigo}

Hodniki, P.P.; Teixeira, C.R.S.; Zanetti, M.L.; Moraes, C.; Fermino, R.C.; Berra, S.

Prática de atividade física e ambiente percebido de usuários do Sistema Único de Saúde

prática de AFD obteve-se que os homens têm mais chance de praticar AFD do que as mulheres (OR 1,62). Quanto à faixa etária os usuários dos serviços públicos de saúde entre 35 a 59 anos (OR 1,85) e de 18 a 34 anos $(\mathrm{OR} 1,99)$ têm mais chance de praticar AFD em relação às pessoas com 60 anos ou mais. As pessoas com classe econômica mais baixa (D e E) $(\mathrm{OR} 2,42)$ e as da classe C (OR 2,01) têm mais chance de praticar AFD comparativamente as pessoas de classe econômica mais alta (A). E os participantes que não souberam ou referiram não ter local para caminhar no bairro onde residem, parecem ter o valor de oportunidades opostas (OR 0,52$)$ chances de serem ativos no lazer (Tabela 4).

Tabela 3 - Modelo de regressão logística entre percepção do ambiente segundo sexo, distância referida entre o domicilio e

Posto de Saúde e feira dos usuários dos serviços de saúde, Ribeirão Preto - SP, 2019

\section{VARIÁVEL}

OR

\section{Atividade Física de Lazer}

\section{Sexo}

Feminino

Masculino

Posto de Saúde

Perto ${ }^{1}$

Longe $^{2}$

Não tem / Não sabe

Feira

Perto

Longe

Não tem / Não sabe
MODELO MÚLTIPLO

IC95\%
P*
$0,0441^{*}$

1,0061

1,1261

2,6514

$0,0130^{*}$

1,7187

0,1762

2,2865

0,6664

Teste realizado: Regressão Logística Modelo Múltiplo; IC95\%=Intervalo de Confiança de 95\%; * $\mathrm{p}<0,005$; OR=Oddsratio; 'Locais perto da residência, considerando uma caminhada de até 10 minutos; ${ }^{2}$ Locais longe da residência, considerando uma caminhada de mais de 10 minutos.Fonte: elaboração própria.

Tabela 4 - Modelo de regressão logística entre percepção do ambiente segundo sexo, idade, classe econômica e local para caminhar dos usuários dos serviços de saúde, Ribeirão Preto - SP, 2019

\section{VARIÁVEL}

\section{Atividade Física de deslocamento}

Sexo

Feminino

Masculino

Idade

60 anos ou mais

35 a 59 anos

18 a 34 anos

Classe Econômica

$A+B$

$C$

$D+E$

Local para caminhar

Perto ${ }^{1}$

Longe $^{2}$
ODDS RATIO

1,6168

1,0833

1,1398

3,0889

3,3815

$0,0148^{*}$

1,1919

1,3157

3,1289

$0,0015^{*}$

2,4211

1,2993

4,4696
$0,0049^{*}$ 


\section{Não tem / Não sabe}

0,5212

0,3106

0,8511

$0,0110^{*}$

Teste realizado: Regressão Logística Modelo Múltiplo; IC95\%=Intervalo de Confiança de 95\%; ${ }^{*} \mathrm{p}<0,005$; OR=Oddsratio; 'Locais perto da residência, considerando uma caminhada de até 10 minutos; ${ }^{2}$ Locais longe da residência, considerando uma caminhada de mais de 10 minutos. Fonte: elaboração pró

\section{DISCUSSÃO}

O desenvolvimento de DCNT está intrisecamente relacionado com os determinantes sociais de saúde, ou seja, o ambiente e as condições de vida impostas a determinadas populações traduzem-se em estilo de vida nocivo a saúde do usuario e de seu coletivo ou inversamente, em um estilo de vida preventivo e promotor de saúde. ${ }^{17}$ A maioria, $71,21 \%$, dos participantes do presente estudo era do sexo feminino, corroborando com outra pesquisa nacional que evidenciou maior utilização dos serviços de saúde do SUS pelas mulheres, o que é justificado por uma maior compreensão da mulher aos sinais e sintomas das doenças e, portanto, maior busca pelo serviço de saúde. ${ }^{18}$

Foi constatado, também, que a maioria $(70,1 \%)$ dos usuários tinha DCNT o que está em consenso com a Pesquisa Nacional de Saúde (PNS), realizada no Brasil, em 2013, na qual foi evidenciado que as pessoas com DCNT utilizam duas vezes mais os serviços do SUS do que as pessoas sem DCNT, devido a alta demanda periódica de avaliação do estado de saúde desses usuários. ${ }^{18}$ Assim como, existe uma falha na disseminação do conhecimento acerca dos serviços oferecidos pelo SUS, fazendo com que os usuários procurem os serviços apenas quando se sentem doentes, e não de forma preventiva, como é previsto no atendimento primário. ${ }^{19} \mathrm{Em}$ concordância aos nossos resultados quanto a ocupação, estudo mostrou que grande parte dos usuários estava empregada ${ }^{20}$. Em contrapartida, um estudo realizado apenas com usuários do sexo masculino constatou que os que mais utilizavam os serviços eram os desempregados. ${ }^{21}$

Com relação às características sociodemográficas que podem interferir no nível de atividade física do indivíduo, grande parte dos participantes tinham ensino fundamental incompleto $(40,7 \%)$ e apenas $14,6 \%$ possuíam ensino supe- rior. Estudo realizado, em 2011, com a população de Ribeirão Preto-SP, evidenciou que a prevalência de prática de AF insuficiente foi elevada entre mulheres de baixa renda e escolaridade. ${ }^{22}$. No Brasil, a prática de AF é proporcionalmente mais elevada em individuos com maior nivel de escolaridade conforme apontato pelo Instituto Brasileiro de Geografia e Estatística (IBGE) e pela Pesquisa Nacional por Amostra de Domicílios (PNAD) de $2015^{23}$. Em referência à classe econômica, a maioria, $51,9 \%$ era da classe C. Um estudo que investigou a prática de $\mathrm{AF}$ e a associação com ambiente, com mulheres que viviam no sul do Brasil, mostrou que as mulheres pertencentes a uma classe econômica alta praticavam mais AF, pois pareciam ter maior acesso à academia e profissionais particulares. ${ }^{24}$ Por outro lado, no nosso estudo, a maioria vive em condições de classe econômica baixa e dependem de locais públicos para a prática

\section{Nosso estudo}

mostrou que

os homens têm

mais chances de

serem fisicamente

ativos do que

as mulheres, tanto

para AFL quanto

para AFD. de AF que estejam em condições favoráveis, como mais áreas verdes próximas à residência, praças e parques.

A relação entre percepção do ambiente e atividade física tem sido mostrada em estudos realizados em diferentes cidades do Brasil, como o realizado na cidade de São Paulo, com homens e mulheres com idade superior a 18 anos, baixa renda e escolaridade, que relacionou a AFL e AFD e fatores individuais e ambientais.

Esse estudo mostrou que receber convites de amigos para atividades, pouca poluição ambiental e segurança são atributos considerados positivos para a prática AF6. Nosso estudo mostrou que os homens têm mais chances de serem fisicamente ativos do que as mulheres, tanto para AFL quanto para AFD. Esses resultados estão em concordância a outros estudos que também investigaram a prática de AF e ambiente. . $^{25,26}$

A idade e a classe social parecem ter forte influência sobre a quantidade de deslocamento ativo realizado. Constatou-se que os participantes idosos (maiores de 60 anos) tem menos chance de ser ativo no deslocamento do que os mais jovens, corroborando com achados de outros estudos que identificaram redução progressiva na realização de atividade fisica quanto ao deslocamento durante o processo de envelhecimento. As razões para essa redução estariam relacionadas às perdas funcionais da capacidade aeróbica, flexibilidade, agilidade, coordenação, além do acometimento por DCNT. ${ }^{27,28}$ Outro aspecto relevante evidenciado foi que as pessoas pertencentes às classes econômicas mais baixas $(C, D$ e E) têm mais chance de realizar AFD em comparação às pessoas das classes econômicas mais altas (A e B).

Esses resultados são compatíveis a aos resultados publicados no Suplemento sobre Saúde da Pesquisa Nacional por Amostra de Domicílios (PNAD), realizada pelo Instituto Brasileiro de 


\section{artigo}

Hodniki, P.P.; Teixeira, C.R.S.; Zanetti, M.L.; Moraes, C.; Fermino, R.C.; Berra, S.

Prática de atividade física e ambiente percebido de usuários do Sistema Único de Saúde

Geografia e Estatísticas (IBGE). Esses resultados mostraram que fatores como precariedade do transporte público e restrição orçamentaria das familias mais pobres em relação à aquisição de meios de transporte são determinantes para pratica de AFD na população mais carente, excetuando, dessa forma, uma prática estimulada pelos benefícios socioambientais e de saúde. ${ }^{29}$

Em um estudo que investigou os fatores do ambiente social e construído de ciclistas residentes dos Estados Unidos, mostrou que os fatores ambientais percebidos em relação a pelo menos um item de acessibilidade foi significativamente associada tanto ao uso de bicicleta no lazer, quanto como meio de deslocamento. E a segurança no trânsito, densidade residencial, conectividade de ruas e infraestrutura foram significativamente associados ao uso de bicicleta como meio de deslocamento. ${ }^{30}$ Outro estudo mostrou que a prática de $\mathrm{AF}$ de pessoas que moram perto ou longe de um centro de intervenção para a prática de $\mathrm{AF}$, como ocorre em alguns postos de saúde do Brasil, que a promoção da prática de $\mathrm{AF}$ é facilitada a medida que o usuário reside mais próximo desses locais. ${ }^{31}$

\section{CONCLUSÃO}

Conclui-se que as intervenções para a promoção de saúde em relação à prática de AF de usuários de serviços do SUS devem considerar a criação e manutenção de ambiente propício para a prática de AFL e AFD como locais para caminhada acessível e acesso a áreas verdes de boa qualidade. Também atentar ao perfil da população para desenvolver políticas públicas de saúde que foquem em ações promotoras de AF direcionadas às mulheres, pessoas com idade mais avançada, classe econômica e escolaridade baixa.

\section{REFERÊNCIAS}

1. Adilson Marques, Miguel Peralta, Hugo Sarmento, João Martins, Miguel González Valeiro, Associations between vigorous physical activity and chronic diseases in older adults: a study in 13 European countries, European Journal of Public Health, Volume 28, Issue 5, October 2018, Pages 950-955, https://doi. org/10.1093/eurpub/cky086

2. Promoting Physical Activity and Exercise: JACC Health Promotion Series. Citation Datajournal of the American College of Cardiology, ISSN: 1558-3597, Vol: 72, Issue: 14, Page: 1622-1639, 2018

3. Bullard, T., Ji, M., An, R. et al. A systematic review and meta-analysis of adherence to physical activity interventions among three chronic conditions: cancer, cardiovascular disease, and diabetes. BMC Public Health 19, 636 (2019). https://doi. org/10.1186/s12889-019-6877-z

4. Vigitel Brasil 2018: vigilância de fatores de risco e proteção para doenças crônicas por inquérito telefônico : estimativas sobre frequência e distribuição sociodemográfica de fatores de risco e proteção para doenças crônicas nas capitais dos 26 estados brasileiros e no Distrito Federal em 2018 / Ministério da Saúde, Secretaria de Vigilância em Saúde, Departamento de Análise em Saúde e Vigilância de Doenças não Transmissíveis. - Brasília: Ministério da Saúde, 2019. 132.: il. Modo de acesso: World Wide Web: http://bvsms.saude.gov.br/bvs/publicacoes/ vigitel_brasil_2018 _vigilancia_fatores_risco.pdf. ISBN 97885-334-2705-1)

5. Ministério da Saúde (BR), Secretaria de Vigilância em Saúde, Departamento de Análise de Situação de Saúde. Plano de ações estratégicas para o enfrentamento das Doenças Crônicas Não Transmissíveis (DCNT) no Brasil 2011-2022. Brasília (DF); 2011 [citado 2018 out 28]. (Série B. Textos Básicos de Saúde). Disponivel em: http://bvsms.saude.gov.br/publicacoes/plano_ acoes_enfrent_dcnt_2011.pdf

6. Florindo AA, Salvador EP, Reis RS, Guimarães VV. Percepção do ambiente e prática de atividade física em adultos residentes em região de baixo nível socioeconômico. Rev Saude Publica. 2011; 45 (2): 302-310. doi: 10.1590/S0034-89102011000200009
7. Devarajan R, Prabhakaran D, Goenka S. Built environment for physical activity-An urban barometer, surveillance, and monitoring. Obes Rev. 2020 Jan; 21 (1): e12938. doi: 10.1111 / obr.12938. Epub 2019 7. de novembro. PMID: 31701653; PMCID: PMC6916279.

8. Silva ZP, Ribeiro MCSA, Barata RB, Almeida MF. Perfil sociodemográfico e padrão de utilização dos serviços de saúde do Sistema Único de Saúde (SUS), 2003- 2008. Cienc saúde coletiva. 2011; 16 ( 9 ): 3807-3816. doi: 10.1590/S141381232011001000016

9. Instituto Brasileiro de Geografia e Estatística. Censo 2010. Rio de Janeiro: IBGE; 2010 [citado 2018 out 20]. Disponível em: https://censo2010.ibge.gov.br/

10. PMRP/SMS - Prefeitura Municipal de Ribeirão Preto/Secretaria Municipal de Saúde. Plano Municipal de Saúde 2018-2021 [Internet]. Ribeirão Preto: SMS/Prefeitura de Ribeirão Preto; 2017 [citado 2018 out 29]. Disponivel em: https://www.ribeiraopreto.sp.gov.br/ssaude/pdf/pms-rp-2018-2021.pdf

11. Ministério da Saúde (BR). Secretaria de Atenção à Saúde. Departamento de Atenção Básica. Diretrizes Nacionais de Implantação da Estratégia e-SUS Atenção básica. [Internet] Brasília (DF); 2014. [citado 2018 dez 10]. Disponível em: http://189.28.128.100/dab/docs/portaldab/publicacoes/diretrizes_nacionais_esus.pdf.

12. Brasil. Critério de Classificação Econômica Brasil/2012 [Internet]. ABEP: Associação Brasileira de Empresas de Pesquisa [citado 2019 jan 20]. Disponivel em: http://www.abep.org/criterio-brasil.

13. Instituto Brasileiro de Geografia e Estatística. Pesquisa Nacional de Saúde: percepção do estado de saúde, estilos de vida e doenças crônicas: 2013 [Internet]. Rio de Janeiro: IBGE; 2014 [citado 2019 jan 20]. Disponível em: ftp://ftp.ibge.gov.br/ PNS/2013/pns2013.pdf

14. Matsudo SM, Matsudo VR, Araújo T, Andrade D, Andrade E, Oliveira LC, et al. Nível de atividade física da população do Es- 


\section{REFERÊNCIAS}

tado de São Paulo: análise de acordo com o gênero, idade, nível sócio-econômico, distribuição geográfica e de conhecimento. Rev Bras de Cien e Mov [Internet]. 2002 [citado 2019 jan 20]; 10: 41-50. Disponivel em: http://cev.org.br/biblioteca/nivel-atividade-fisica-populacao-estado-sao-paulo-analise-acordo-com-o-genero-idade-nivel-socioeconomico-distribuicao-geografica-de-conhecimento.

15. Malavasi LDM, Duarte MFS, Both J, Reis RS. Escala de mobilidade ativa no ambiente comunitário - NEWS Brasil: retradução e reprodutibilidade. Rev Bras Cineantropom Desempenho Hum [Internet]. 2007 [citado 2019 jan 20]; 9(4): 339-50. Disponivel em: http://ipenproject.org/documents/publications_docs/Reis_ Brazilian_NEWS.pdf.

16. R Core Team. R: A language and environment for statistical computing. R Foundation for Statistical Computing [Internet]. 2013. Vienna, Austria; 2013 [citado 2019 jan 30]. Disponivel em: http://www.R-project.org/.

17. Malta DC, Merhy EE. O percurso da linha do cuidado sob a perspectiva das doenças crônicas não transmissíveis. Interface (Botucatu) [Internet]. 2010 [citado 2019 jan 30]; 14( 34 ): 593606. Disponivel em: http://www.scielo.br/scielo.php?script=sci_arttext\&pid=S1414-32832010000300010\&lng=en. doi: 10.1590/S1414-32832010005000010.

18. Malta DC, Bernal RTI, Lima MG, Araújo SSC, Silva MMA, Freitas MIF et al . Doenças crônicas não transmissíveis e a utilização de serviços de saúde: análise da Pesquisa Nacional de Saúde no Brasil. Rev. Saude Publica [Internet]. 2017 [citado 2019 fev 20]; 51(Suppl 1): 45. Disponível em: http://www.scielo.br/scielo. php?script=sci_arttext\&pid=S0034-89102017000200306\&lng=en. doi: 10.1590/s1518-8787.2017051000090.

19. Hamada RKF, de Almeida VOC, Brasil ICF, Souza SG, Luzia RAB, Campos EMS, Leite ICG. Conhecendo o Sistema Único de Saúde: um olhar da população. Rev de APS [Internet]. 2018 [citado 2020 fev 21]; 21(4): 504-515. Disponivel em: https://periodicos.ufjf.br/index.php/aps/article/view/16459. doi: https://doi. org/10.34019/1809-8363.2018.v21.16459.

20. Moimaz SAS, Lima AMC, Garbin CAS, Corrente JE, Saliba NA. Avaliação do usuário sobre o atendimento odontológico no Sistema Único de Saúde: uma abordagem à luz da humanização. Cienc. saude coletiva [Internet]. 2016 [citado 2019 fev 21]; 21(12 ): 3879-3887. Disponível em: http://www.scielo.br/scielo. php?script=sci_arttext\&pid=S1413-81232016001203879\&Ing=en. doi: 10.1590/1413-812320152112.12672015.

21. Arruda GO, Mathias TAF, Marcon SS. Prevalência e fatores associados à utilização de serviços públicos de saúde por homens adultos. Ciênc. saúde coletiva [Internet]. 2017 [citado 2019 fev 21]; 22(1): 279-290. Disponivel em: http://www.scielo.br/scielo. php?script=sci_arttext\&pid $=$ S1413-81232017000100279\& $\mathrm{I}$ ng=en. doi: 10.1590/1413-81232017221.20532015.

22. Suzuki CS, Moraes SA, Freitas ICM. Atividade física e fatores associados em adultos residentes em Ribeirão Preto, SP. Rev Saude Publica [Internet]. 2011 [citado 2019 jan 24]; 45(2): 311-320. Disponivel em: http://www.scielo.br/scielo.
php?script=sci_arttext\&pid=S0034-89102011000200010\&Ing=en. doi: 10.1590/S0034-89102011000200010.

23. Instituto Brasileiro de Geografia e Estatística. Práticas de esporte e atividade física: 2015 [Internet]. Rio de Janeiro: IBGE; 2017 [citado 2019 fev 24]. Disponível em: https://biblioteca.ibge. gov.br/visualizacao/livros/liv100364.pdf

24. Boing L, Guimarães ACA, Araujo CCR, Bertuol C, Hammes JF, Pazin J, et al. Physical activity associated with urban environmental characteristics: A correlational study of active women of high socioeconomic status from Brazilian cities of Santa Catarina. Motriz. 2015;21 (4):393-402. doi: 10.1590/S198065742015000400008.

25. Bauman AE, Reis RS, Sallis JF, Wells JC, Loos RJ, Martin BW. Correlates of physical activity: why are some people physically active and others not? Lancet. 2012; 380 (9838):258-271. doi: 10.1016/S0140-6736(12)60735-1.

26. Turi BC, Codogno JS, Fernandes RA, Lynch KR, Kokubun E, Monteiro HL. Time trends in physical activity of adult users of the Brazilian National Health System: 2010-2014. Longitudinal study. Sao Paulo Med J [Internet]. 2017 [citado 2019 fev 26]; 135(4): 369-375. Disponivel em: http://www.scielo.br/scielo. php?script=sci_arttext\&pid=S1516-31802017000400369\&lng=en. doi: 10.1590/1516-3180.2017.0025190317.

27. Peixoto SV, Mambrini JVM, Firmo JOA, Loyola Filho Al, Souza JPRB, Andrade FB et al. Prática de atividade física entre adultos mais velhos: resultados do ELSI-Brasil. Rev Saude Publica [Internet]. 2018 [citado 2019 abr 6]; 52(Suppl 2): 5s. Disponivel em: http://www.scielo.br/scielo.php?script=sci_arttext\&pid=S0034-89102018000300501\&lng=en. doi: 10.11606/ s1518-8787.2018052000605.

28. Krug RR, Lopes MA, Balbé GP, Marchesan M, Mazo GZ. Prevalence of commuting physical activity and associated factors in long-lived older adults. Rev bras cineantropom desempenho hum [Internet]. 2016 [citado 2019 abr 6]; 18(5): 520-529. Disponivel em: http://www.scielo.br/scielo. php?script=sci_arttext\&pid=S1980-00372016000500520\&lng=en. doi: 10.5007/1980-0037.2016v18n5p520.

29. Sá TH, Pereira RHM, Duran AC, Monteiro CA. Diferenças socioeconômicas e regionais na prática do deslocamento ativo no Brasil. Rev Saude Publica [Internet]. 2016 [citado 2019 abr 8]; 50:37. Disponivel em: http://www.scielo.br/scielo.php?script=sci_arttext\&pid=S0034-89102016000100222\&Ing=en. doi: $10.1590 / \mathrm{S} 1518-8787.2016050006126$.

30. Porter AK, Kohl HW, Pérez A, Reininger B, Pettee Gabriel K, Salvo D. Perceived Social and Built Environment Correlates of Transportation and Recreation-Only Bicycling Among Adults. Prev Chronic Dis. 2018; 15:E135. doi:10.5888/pcd15.180060

31. Andrade ACS, Mingoti SA, Fernandes AP, Andrade RG, Friche $A A L$, Xavier CC et al. Neighborhood-based physical activity differences: Evaluation of the effect of health promotion program. PLoS ONE. 2018; 13(2): e0192115. doi:10.1371/journal. pone.0192115 